Pacific Journal of Mathematics

SEQUENCES IN GROUPS WITH DISTINCT PARTIAL 


\section{SEQUENCES IN GROUPS WITH DISTINCT \\ PARTIAL PRODUCTS}

\section{BASIL GoRDON}

1. In an investigation concerning a certain type of Latin square, the following problem arose:

Can the elements of a finite group $G$ be arranged in a sequence $a_{1}, a_{2}, \cdots, a_{n}$ so that the partial products $a_{1}, a_{1} a_{2}, \cdots, a_{1} a_{2} \cdots a_{n}$ are all distinct?

In the present paper a complete solution will be given for the case of Abelian groups, and the application to Latin squares will be indicated. Let us introduce the term sequenceable group to denote groups whose elements can be arranged in a sequence with the property described above. The main result is then contained in the following theorem.

THEOREM 1. A finite Abelian group $G$ is sequenceable if and only if $G$ is the direct product of two groups $A$ and $B$, where $A$ is cyclic of order $2^{k}(k>0)$, and $B$ is of odd order.

Proof (i). To see the necessity of the condition, suppose that $G$ is sequenceable, and let $a_{1}, a_{2}, \cdots, a_{n}$ be an ordering of the elements of $G$ with $a_{1}, a_{1} a_{2}, \cdots, a_{1} a_{2} \cdots a_{n}$ all distinct. The notation $b_{i}=a_{1} a_{2} \cdots a_{i}$ will be used throughout the remainder of the paper. It is immediately seen that $a_{1}=b_{1}=e$, the identity element of $G$; for if $a_{i}=e$ for some $i>1$, then $b_{i-1}=b_{i}$, contrary to assumption. Hence $b_{n} \neq e$, i.e., the product of all the elements of $G$ is not the identity. It is well known (cf [2]) that this implies that $G$ has the form $A \times B$ with $A$ cyclic of order $2^{k}(k>0)$ and $B$ of odd order.

(ii) To prove sufficiency of the condition, suppose that $G=A \times B$, with $A$ and $B$ as above. We then show that $G$ is sequenceable by constructing an ordering $a_{1}, a_{2}, \cdots, a_{n}$ of its elements with distinct partial products. From the general theory of Abelian groups, it is known that $G$ has a basis of the form $c_{0}, c_{1}, \cdots, c_{m}$, where $c_{0}$ is of order $2^{k}$, and where the orders $\delta_{1}, \delta_{2}, \cdots, \delta_{m}$ of $c_{1}, c_{2}, \cdots, c_{m}$ are odd positive integers each of which divides the next, i.e., $\delta_{i} \mid \delta_{i+1}$ for $0<i<m$. If $j$ is any positive integer, then there exist unique integers $j_{0}, j_{1}, \cdots, j_{m}$ such that

$$
\begin{aligned}
j & \equiv j_{0}\left(\bmod \delta_{1} \delta_{2} \cdots \delta_{m}\right) \\
j_{0} & =j_{1}+j_{2} \delta_{1}+j_{3} \delta_{1} \delta_{2}+\cdots+j_{m} \delta_{1} \cdots \delta_{m-1} \\
& 0 \leqq j_{1}<\delta_{1}
\end{aligned}
$$

Received January 3, 1961. 


$$
\begin{aligned}
& 0 \leqq j_{2}<\delta_{2} \\
& \vdots \\
& 0 \leqq j_{m}<\delta_{m} .
\end{aligned}
$$

The proof of the existence and uniqueness of this expansion will be omitted here; it is entirely analogous to the expansion of an integer in powers of a number base.

We are now in a position to define the desired sequencing of $G$. It is convenient to define the products $b_{1}, b_{2}, \cdots, b_{n}$ directly, to prove they are all distinct, and then to verify that the corresponding $a_{i}$, as calculated from the formula $a_{1}=e, a_{i}=b_{i-1}^{-1} b_{i}$, are all distinct. If $i$ is of the form $2 j+1(0 \leqq j<n / 2)$, let

$$
b_{2 j+1}=c_{0}^{-j} c_{1}^{-j_{1}} c_{2}^{-j_{2}} \cdots c_{m}^{-j_{m}},
$$

where $j_{1}, j_{2}, \cdots, j_{m}$ are the integers defined in (1). On the other hand, if $i$ is of the form $2 j+2(0 \leqq j<n / 2)$, let

$$
b_{2 j+2}=c_{0}^{j+1} c_{1}^{j_{1}+1} c_{2}^{j_{2}+1} \cdots c_{m}^{j_{m}+1} .
$$

The elements $b_{1}, b_{2}, \cdots, b_{n}$ thus defined are all distinct. For if $b_{s}=b_{t}$ with $s=2 u+1, t=2 v+1$, then

$$
\begin{gathered}
u \equiv v\left(\bmod 2^{k}\right) \\
u_{1} \equiv v_{1}\left(\bmod \delta_{1}\right) \\
\vdots \\
u_{m} \equiv v_{m}\left(\bmod \delta_{m}\right) .
\end{gathered}
$$

From the inequalities in (1) we conclude that $u_{1}=v_{1}, \cdots, u_{m}=v_{m}$. Hence $u_{0}=v_{0}$, so that $u \equiv v\left(\bmod \delta_{1} \cdots \delta_{m}\right)$; coupled with the first of equations (2), this gives $u \equiv v(\bmod n)$, which implies $u=v$. Similarly $b_{2 u+2}=b_{2 v+2}$ implies $u=v$, so that the "even" $b$ 's are distinct.

Next suppose

$$
b_{2 u+1}=b_{2 v+2} \text {. }
$$

Then

$$
\begin{aligned}
& -u \equiv v+1\left(\bmod 2^{k}\right) \\
& -u_{1} \equiv v_{1}+1\left(\bmod \delta_{1}\right) \\
& \vdots \\
& -u_{m} \equiv v_{m}+1\left(\bmod \delta_{m}\right)
\end{aligned}
$$

or equivalently,

$$
u+v+1 \equiv 0\left(\bmod 2^{k}\right)
$$




$$
\begin{gathered}
u_{1}+v_{1}+1 \equiv 0\left(\bmod \delta_{1}\right) \\
\vdots \\
u_{m}+v_{m}+1 \equiv 0\left(\bmod \delta_{m}\right) .
\end{gathered}
$$

Since $0<u_{1}+v_{1}+1 \leqq 2\left(\delta_{1}-1\right)+1<2 \delta_{1}$, we must have $u_{1}+v_{1}+1=$ $\delta_{1}$. Reasoning similarly for $i=2, \cdots, m$ we obtain

$$
\begin{aligned}
& u_{1}+v_{1}+1=\delta_{1} \\
& u_{2}+v_{2}+1=\delta_{2} \\
& \vdots \\
& u_{m}+v_{m}+1=\delta_{m} .
\end{aligned}
$$

Multiplying the $(i+1)$ 'st equation of this system by $\delta_{1} \delta_{2} \cdots \delta_{i}(1 \leqq i<m)$ and adding, we get $u_{0}+v_{0}+1=\delta_{1} \cdots \delta_{m}$, which implies $u+v+1 \equiv$ $o\left(\delta_{1} \cdots \delta_{m}\right)$. Combining this with the first of equations (3), we find that $u+v+1 \equiv 0(\bmod n)$, which, on account of the inequality $0<u+v+$ $1<n$, is impossible. Hence $b_{1}, b_{2}, \cdots, b_{n}$ are all distinct.

Next we calculate $a_{1}, a_{2}, \cdots, a_{n}$. If $i=2 j+2(0 \leqq j<n / 2)$, then

$$
a_{i}=b_{i-1}^{-1} b_{i}=c_{0}^{2 j+1} c_{1}^{2 j_{1}+1} \cdots c_{m}^{2 j_{m}+1} .
$$

These are all different by the same argument as above. If $i=2 j+1$, and $j_{1} \neq 0$, then

$$
a_{i}=c_{0}^{-2 j} c_{1}^{-2 \jmath_{1}} c_{2}^{-2 j_{2}-1} \cdots c_{m}^{-2 \jmath_{m}-1} .
$$

If $i=2 j+1$ and $j_{1}=0$, but $j_{2} \neq 0$, then $a_{i}=c_{0}^{-2 j} c_{2}^{-2 j_{2}} c_{3}^{-2 j_{3}-1} \cdots c_{m}^{-2 j_{m}-1}$, while if $j_{1}=j_{2}=0$ but $j_{3} \neq 0$, then $a_{i}=c_{0}^{-2 j} c_{3}^{-2 j_{3}} c_{4}^{-2 j_{4}-1} \cdots c_{m}^{-2 j_{m}-1}$, etc.

\begin{tabular}{|c|c|c|c|}
\hline \multicolumn{2}{|c|}{$a_{i}$} & \multicolumn{2}{|c|}{$b_{i}$} \\
\hline$\left(\begin{array}{ll}0 & 0\end{array}\right.$ & 0 ) & $(0$ & 0) \\
\hline (1 & 1) & (1 & 1) \\
\hline (0 & 2) & (1 & 0) \\
\hline (1 & 1) & (0 & 1) \\
\hline (0 & 2) & (0 & 0) \\
\hline (1 & 1) & (1 & 1) \\
\hline$(0$ & 1) & (1 & 2) \\
\hline (1) & $10)$ & (0) & 2) \\
\hline
\end{tabular}
These $a_{i}$ 's are obviously distinct from each other by the same reasoning as before. Because of the exponent of $c_{0}$ they are also distinct from the $a_{i}$ with $i$ even. This completes the proof of the theorem.

As an example of the construction of Theorem 1, consider the group $G=C_{2} \times C_{3} \times C_{3}$. We use basis elements $c_{0}, c_{1}, c_{2}$ of orders $2,3,3$ respectively. Using the notation $(\alpha, \beta, \gamma)$ for the element $c_{0}^{\alpha} c_{1}^{\beta} c_{2}^{\gamma}$, the sequences $a_{i}$ and $b_{i}$ are then the following: 


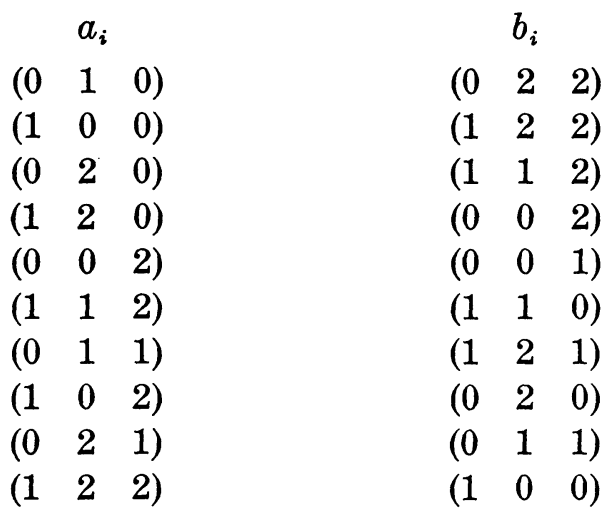

2. Application to Latin squares. Consider the following Latin square:

$\begin{array}{llll}1 & 2 & 3 & 4 \\ 2 & 4 & 1 & 3 \\ 3 & 1 & 4 & 2 \\ 4 & 3 & 2 & 1\end{array}$

Given any ordered pair $(\alpha \beta)$ with $\alpha \neq \beta$, it occurs as a pair of consecutive entries in some row of this square. In general, an $n \times n$ Latin square $\left(c_{s t}\right)$ whose elements are the integers $1, \cdots, n$ will be called horizontally complete if for every ordered pair $(\alpha, \beta)$ with $1 \leqq \alpha, \beta \leqq n$ and $\alpha \neq \beta$, the equations

$$
\begin{aligned}
c_{s t} & =\alpha \\
c_{s, t+1} & =\beta
\end{aligned}
$$

are solvable. Similarly a vertically complete square is one for which

$$
\begin{aligned}
c_{s t} & =\alpha \\
c_{s+1, t} & =\beta
\end{aligned}
$$

can be solved for any such choice of $\alpha, \beta$. A square which is both horizontally and vertically complete is called complete.

Note that in a horizontally complete square, the solution of equations (4) is unique, since the total number of consecutive pairs $a_{s t}, a_{s, t+1}$ is equal to the total number of order pairs $(\alpha, \beta)$ with $\alpha \neq \beta$. Conversely, uniqueness implies existence for the same reason.

Complete Latin squares are useful in the design of experiments in which it is desired to investigate the interaction of nearest neighbors.

THEOREM 2. Suppose that $G$ is a sequenceable group, and let $a_{1}$, $a_{2} \cdots, a_{n}$ be an ordering of its elements such that $b_{1}, b_{2}, \cdots, b_{n}$ are distinct. Then the matrix $\left(c_{s t}\right)=\left(b_{s}^{-1} b_{t}\right)$ is a complete Latin square. 
Proof. It is immediately seen that $\left(c_{s t}\right)$ is a Latin square, since either $b_{s}^{-1} b_{t}=b_{s}^{-1} b_{u}$ or $b_{t}^{-1} b_{s}=b_{u}^{-1} b_{s}$ imply $t=u$ by elementary properties of groups. To show that $\left(c_{s t}\right)$ is horizontally complete, suppose

$$
\begin{aligned}
c_{s t} & =c_{u v} \\
c_{s, t+1} & =c_{u, v+1} .
\end{aligned}
$$

We must show that $s=u$ and $t=v$. From the definition of $c_{s t}$,

$$
\begin{aligned}
b_{s}^{-1} b_{t} & =b_{u}^{-1} b_{v} \\
b_{s}^{-1} b_{t+1} & =b_{u}^{-1} b_{v+1} .
\end{aligned}
$$

Inverting both sides of (5) yields $b_{t}^{-1} b_{s}=b_{u}^{-1} b_{u}$. Combining this with (6) we get $\left(b_{t}^{-1} b_{s}\right)\left(b_{s}^{-} b_{t+1}\right)=\left(b_{v}^{-1} b_{u}\right)\left(b_{u}^{-1} b_{v+1}\right)$, or $b_{t}^{-1} b_{t+1}=b_{v}^{-1} b_{v+1}$, i.e., $a_{t+1}=a_{v+1}$. This implies $t=v$. Substituting in (5) we obtain $b_{s}^{-1} b_{t}=b_{u}^{-1} b_{t}$, from which $s=u$ follows immediately. The proof that $\left(c_{s t}\right)$ is vertically complete is entirely similar and will be omitted.

This method enables one to construct a complete Latin square of order $n$ for any even $n$ (note that $B$ may be trivial in Theorem 1). Whether or not complete, or even horizontally complete, squares exist for odd $n$ is an open question.

3. Extension to non-Abelian groups. The problem of determining which non-Abelian groups $G$ are sequencable is unsolved at the present time. Considerable information about the nature of a sequence $a_{1}, \cdots, a_{n}$ with distinct partial products, if one exists, can be obtained by mapping $G$ onto the Abelian group $G / C$, where $C$ is the commutator subgroup. Using this technique, for example, it can be shown that the non-Abelian group of order 6 and the two non-Abelian groups of order 8 are not sequencable. On the other hand the non-Abelian group of order 10 is sequencable. To see this, denote its elements by $e, a, b, a b, b a, a b a, b a b$, $a b a b, b a b a, a b a b a$, where $a^{2}=b^{2}=(a b)^{5}=e . \quad$ A suitable ordering is then given by $e, a b, a b a b, a b a b a, b a b, a b a, b, a, b a b a, b a$, the partial products being $e, a b, b a b a, a, a b a b, b a b, b a, b, a b a, a b a b a$. In view of Theorem 1 and the results of [2], one might conjecture that $G$ is sequencable if and only if it does not possess a complete mapping. However, the symmetric group $S_{3}$ does not possess a complete mapping (cf [1]) and is also not sequenceable. Whether or not the two properties are at least mutually exclusive is still an open question.

\section{REFERENCES}

1. L. J. Paige, Complete mappings of finite groups, Pacific J. Math. 1 (1951), 111-116.

2. M. Hall and L. J. Paige, Complete mappings of finite groups, Pacific J. Math. 5 (1955), 541-549. 



\section{PACIFIC JOURNAL OF MATHEMATICS}

\section{EDITORS}

\author{
RaLPh S. Phillips \\ Stanford University \\ Stanford, California \\ F. H. BRowNELL \\ University of Washington \\ Seattle 5 , Washington
}

A. L. Whiteman

University of Southern California

Los Angeles 7, California

L. J. Paige

University of California

Los Angeles 24, California

\author{
E. F. BECKENBACH \\ T. M. CHERRY
}

\author{
ASSOCIATE EDITORS

$\begin{array}{lll}\text { D. DERRY } & \text { H. L. ROYDEN } & \text { E. G. STRAUS } \\ \text { M. OHTSUKA } & \text { E. SPANIER } & \text { F. WOLF }\end{array}$

\section{SUPPORTING INSTITUTIONS}

\author{
UNIVERSITY OF BRITISH COLUMBIA \\ CALIFORNIA INSTITUTE OF TECHNOLOGY \\ UNIVERSITY OF CALIFORNIA \\ MONTANA STATE UNIVERSITY \\ UNIVERSITY OF NEVADA \\ NEW MEXICO STATE UNIVERSITY \\ OREGON STATE COLLEGE \\ UNIVERSITY OF OREGON \\ OSAKA UNIVERSITY \\ UNIVERSITY OF SOUTHERN CALIFORNIA
}

\author{
STANFORD UNIVERSITY \\ UNIVERSITY OF TOKYO \\ UNIVERSITY OF UTAH \\ WASHINGTON STATE COLLEGE \\ UNIVERSITY OF WASHINGTON \\ AMERICAN MATHEMATICAL SOCIETY \\ CALIFORNIA RESEARCH CORPORATION \\ HUGHES AIRCRAFT COMPANY \\ SPACE TECHNOLOGY LABORATORIES \\ NAVAL ORDNANCE TEST STATION
}

Mathematical papers intended for publication in the Pacific Journal of Mathematics should be typewritten (double spaced), and the author should keep a complete copy. Manuscripts may be sent to any one of the four editors. All other communications to the editors should be addressed to the managing editor, L. J. Paige at the University of California, Los Angeles 24, California.

50 reprints per author of each article are furnished free of charge; additional copies may be obtained at cost in multiples of 50 .

The Pacific Journal of Mathematics is published quarterly, in March, June, September, and December. The price per volume (4 numbers) is $\$ 12.00$; single issues, $\$ 3.50$. Back numbers are available. Special price to individual faculty members of supporting institutions and to individual members of the American Mathematical Society: $\$ 4.00$ per volume; single issues, $\$ 1.25$.

Subscriptions, orders for back numbers, and changes of address should be sent to Pacific Journal of Mathematics, 103 Highland Boulevard, Berkeley 8, California.

Printed at Kokusai Bunken Insatsusha (International Academic Printing Co., Ltd.), No. 6, 2-chome, Fujimi-cho, Chiyoda-ku, Tokyo, Japan.

\section{PUBLISHED BY PACIFIC JOURNAL OF MATHEMATICS, A NON-PROFIT CORPORATION}

The Supporting Institutions listed above contribute to the cost of publication of this Journal, but they are not owners or publishers and have no responsibility for its content or policies.

Reprinted 1966 in the United States of America 


\section{Pacific Journal of Mathematics}

\section{Vol. 11, No. 4}

A. V. Balakrishnan, Prediction theory for Markoff processes . . . . . . . . . . 1171

Dallas O. Banks, Upper bounds for the eigenvalues of some vibrating systems . . . . 1183

A. Białynicki-Birula, On the field of rational functions of algebraic groups ...... 1205

Thomas Andrew Brown, Simple paths on convex polyhedra .............. 1211

L. Carlitz, Some congruences for the Bell polynomials . . . . . . . . . . . . 1215

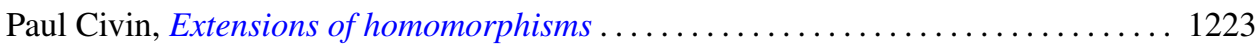

Paul Joseph Cohen and Milton Lees, Asymptotic decay of solutions of differential

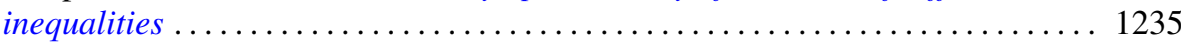

István Fáry, Self-intersection of a sphere on a complex quadric . . . . . . . . . . 1251

Walter Feit and John Griggs Thompson, Groups which have a faithful representation

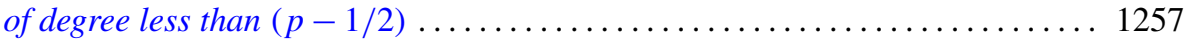

William James Firey, Mean cross-section measures of harmonic means of convex

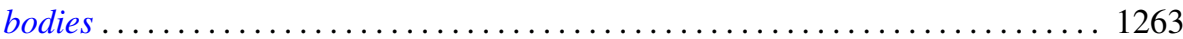

Avner Friedman, The wave equation for differential forms . . . . . . . . . . 1267

Bernard Russel Gelbaum and Jesus Gil De Lamadrid, Bases of tensor products of

Banach spaces ................................... 1281

Ronald Kay Getoor, Infinitely divisible probabilities on the hyperbolic plane . . . . 1287

Basil Gordon, Sequences in groups with distinct partial products . . . . . . . . . . . . 1309

Magnus R. Hestenes, Relative self-adjoint operators in Hilbert space . . . . . . . . . 1315

Fu Cheng Hsiang, On a theorem of Fejér ......................... 1359

John McCormick Irwin and Elbert A. Walker, On N-high subgroups of Abelian

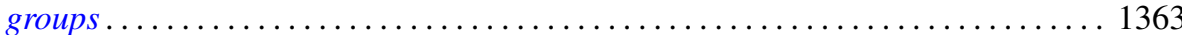

John McCormick Irwin, High subgroups of Abelian torsion groups . . . . . . . . . 1375

R. E. Johnson, Quotient rings of rings with zero singular ideal . . . . . . . . . . . 1385

David G. Kendall and John Leonard Mott, The asymptotic distribution of the time-to-escape for comets strongly bound to the solar system ...

Kurt Kreith, The spectrum of singular self-adjoint elliptic operators ....

Lionello Lombardi, The semicontinuity of the most general integral of the calculus of variations in non-parametric form ................................

Albert W. Marshall and Ingram Olkin, Game theoretic proof that Chebyshev inequalities are sharp

Wallace Smith Martindale, III, Primitive algebras with involution . . William H. Mills, Decomposition of holomorphs ..............

James Donald Monk, On the representation theory for cylindric algebras . . . . . . 1447

Shu-Teh Chen Moy, A note on generalizations of Shannon-McMillan theorem . . . . 1459

Donald Earl Myers, An imbedding space for Schwartz distributions . .

John R. Myhill, Category methods in recursion theory .........

Paul Adrian Nickel, On extremal properties for annular radial and circular slit mappings of bordered Riemann surfaces

Edward Scott O'Keefe, Primal clusters of two-element algebras . .

Nelson Onuchic, Applications of the topological method of Wazewski to certain

problems of asymptotic behavior in ordinary differential equations ...

Peter Perkins, A theorem on regular matrices................

Clinton M. Petty, Centroid surfaces .... 